\title{
Cardiovascular magnetic resonance tagging imaging correlates with myocardial dysfunction and T2 mapping in idiopathic dilated cardiomyopathy
}

\author{
Atsushi K. Kono • Pierre Croisille • Tatsuya Nishii • \\ Koya Nishiyama $\cdot$ Katsusuke Kyotani $\cdot$ Mayumi Shigeru • \\ Sachiko Takamine $\cdot$ Sei Fujiwara $\cdot$ Kazuro Sugimura
}

Received: 16 July 2014/ Accepted: 15 August 2014/Published online: 26 August 2014

(C) Springer Science+Business Media Dordrecht 2014

\begin{abstract}
To evaluate the details of myocardial dysfunction in dilated cardiomyopathy (DCM) patients using tagging images and the correlation of tagging imaging with tissue characteristics. Circumferential strain (Ecc) derived from tagging images was measured in 15 normal (NML) subjects (15 males; mean age 28.5 years) and 12 DCM patients ( 7 males; mean age 48.9 years). The following parameters were compared: (1) the magnitude of peak Ecc (Ecc*); (2) the coefficient of variation of the time of Ecc* (CVtime*), which indexes dyssynchrony; and (3) descriptive findings of time-Ecc curves. We also evaluated the
\end{abstract}

A. K. Kono $(\square)$

Department of Radiology, Kobe University Hospital,

7-5-2 Kusunoki-cho, Chuo-ku, Kobe, Hyogo 650-0017, Japan

e-mail: ringonotegami@mac.com

\section{A. K. Kono}

Department of Radiology, Erasmus Medical Center, 's

Gravendijkwal 230, 3015 CE Rotterdam, The Netherlands

\author{
A. K. Kono $\cdot$ T. Nishii $\cdot$ K. Sugimura \\ Department of Radiology, Kobe University Graduate School \\ of Medicine, 7-5-2 Kusunoki-cho, Chuo-ku, Kobe, \\ Hyogo 650-0017, Japan \\ P. Croisille \\ Department of Radiology, Université Jean Monnet \\ Saint-Etienne, CREATIS, UMR CNRS 5220-INSERM U1044, \\ Lyon, France \\ K. Nishiyama $\cdot$ K. Kyotani \\ Division of Radiology, Kobe University Hospital, 7-5-2 \\ Kusunoki-cho, Chuo-ku, Kobe, Hyogo 650-0017, Japan \\ M. Shigeru $\cdot$ S. Takamine $\cdot$ S. Fujiwara \\ Division of Cardiovascular and Respiratory Medicine, \\ Department of Internal Medicine, Kobe University Graduate \\ School of Medicine, 7-5-2 Kusunoki-cho, Chuo-ku, \\ Kobe 650-0017, Hyogo, Japan
}

correlations of Ecc* in DCM patients with ejection fraction $(\mathrm{EF})$, myocardial $\mathrm{T} 2$ values, and late gadolinium enhancement (LGE). Mean Ecc*s in DCM patients and NML subjects were -12.7 and $-23.5 \%$, respectively $(P<0.0001)$. Mean CVtime*s were 15.2 and $4.5 \%$, respectively $(P=0.0002)$. The findings of pre-systolic extension and systolic stretch in the septum were observed in $6(50 \%)$ and $10(83.3 \%)$ DCM patients and in none of the NML participants. Ecc* was correlated with EF $\left(P<0.0001, R^{2}=0.90\right)$ and T2 values $(P=0.018$, $\left.R^{2}=0.44\right)$ but not with LGE $\left(P=0.072, R^{2}=0.28\right)$. Tagging images revealed the reduction of myocardial function as well as dyssynchrony in DCM patients. Myocardial dysfunction occurred coincidently with myocardial inflammation.

Keywords Strain · Tagging - Dilated cardiomyopathy · Dyssynchrony $\cdot \mathrm{T} 2$ mapping

\section{Introduction}

The use of tagging imaging on cardiovascular magnetic resonance (CMR) images allows the evaluation of the dynamic deformation of lines or grids superimposed on the myocardium during the cardiac cycle. Conventionally, the analysis of left ventricular (LV) function is based on tracing the contour on cine images, which provides information regarding wall motion and wall thickening. In contrast, tagging imaging details the intra-myocardial movement. Radial, circumferential, and longitudinal movement of the myocardium, as well as torsion and rotation of the heart, can be evaluated qualitatively and quantitatively with tagging imaging. Strain, which is expressed as the fractional change in length from the 
resting state to the contractile state, can also be measured [1]. Zerhouni et al. [2] first introduced tagging imaging, and subsequent studies have reported its usefulness in myocardial diseases such as ischemic heart disease [3-5]. Strain is considered a sensitive indicator for the detection of a myocardial infarction and a predictor of myocardial viability. The tagging image is also expected to reveal the physiological and pathological conditions of the myocardium in cardiomyopathy.

Dilated cardiomyopathy (DCM) is the most common form of primary cardiomyopathy. It leads to progressive symptomatic heart failure and major adverse cardiac events including sudden death $[6,7]$. Left ventricular enlargement and systolic dysfunction are well-known pathophysiological findings. CMR has recently been recognized as a useful tool for the diagnosis of DCM because it can detect myocardial edema or inflammation by $\mathrm{T} 2$ weighted image (T2WI) [8] as well as myocardial fibrosis using late gadolinium enhancement (LGE) [9, 10]. LGE makes it possible to distinguish DCM from other cardiomyopathies such as ischemic cardiomyopathy and can also predict its prognosis and adverse cardiac events.

Only a few reports have been published describing tagging imaging in DCM [11-16]. Strain abnormalities previously reported are summarized as follows: the magnitude of the strain, including circumferential strain and myocardial twisting, is reduced; the magnitude of the strain as well as the timing of the contraction shows heterogeneity in space; and abnormal wall motion such as paradoxical contraction in the septum is observed in DCM. The purpose of this study was to evaluate the findings of tagging imaging in DCM patients in order to understand their physiological and pathological conditions and to compare tagging imaging with T2 mapping and LGE imaging.

\section{Materials and methods}

Our Institutional Review Board (Kobe University Graduate School of Medicine) approved this retrospective study and waived written informed consent for the use of patients' clinical and imaging data. We obtained written informed consent from normal controls and informed all patients about the study.

\section{Study population}

We studied 15 normal controls (NML; 15 males, mean age 28.5 years) and 12 DCM patients (5 females, 7 males, mean age 48.9 years). NML group underwent CMR examinations to generate a normal database prior to the study. The diagnosis of DCM was made on the basis of clinical, echocardiographic, and nuclear medicine findings and using myocardial biopsy if necessary. DCM patients fulfilled the criteria reported by Elliot et al. [17]. We excluded patients who showed ischemic cardiomyopathy, arrhythmia, severe anemia requiring frequent blood transfusion, congenital heart disease, history of myocarditis, sarcoidosis, heavy alcohol consumption, adriamycin treatment, muscle disorders such as myositis or muscular dystrophy, or general contraindications for CMR and contrast material.

\section{MRI acquisition}

We used a 3.0-T scanner (Achieva; Philips Healthcare, Best, The Netherlands) with a 6-channel coil for NML group between May and July 2012 and a 1.5-T scanner (Achieva; Philips Healthcare, Best, The Netherlands) with a 5-channel cardiac coil for DCM patients between July 2012 and June 2013. In DCM patients, cine imaging, T2 mapping, and LGE were used in addition to tagging imaging. We used a constant level appearance algorithm (CLEAR), which is a homogeneity correction that compensates for signal inhomogeneity attributable to the surface coils.

\section{Tagging imaging}

Three short-axis images, located at 25,50 and $75 \%$ of the left ventricle, were obtained, in addition to 2- and 4-chamber long-axis images. The slice thickness was $7 \mathrm{~mm}$. The cardiac phase was set at 21 phases. Tag spacing was $6 \mathrm{~mm}$. The NML sequence was a turbo-field echo (TFE) sequence with spatial modulation of the magnetization (SPAMM). The parameters were as follows: field of view (FOV), $250 \mathrm{~mm}$; matrix, $224 \times 224$; echo time (TE), $2.8 \mathrm{~ms}$; repetition time (TR), $4.8 \mathrm{~ms}$ automatically determined by the MR computer; flip angle (FA), $10^{\circ}$; and bandwidth (BW), $434 \mathrm{~Hz}$. The DCM sequence was an echo-planer imaging (EPI) sequence with SPAMM. The parameters were as follows: FOV, $250 \mathrm{~mm}$; matrix, $176 \times 176$; TE, $4.0 \mathrm{~ms}$; TR, $17 \mathrm{~ms}$ automatically determined by the MR computer; FA, 13; and BW, $446 \mathrm{~Hz}$.

\section{Cine imaging}

A total of $12-15$ short-axis cine images, which covered the entire ventricles, were obtained with a thickness of 8 and 2-mm gaps. We also took 2- and 4-chamber long-axis cine images. The parameters for cine imaging included a steady-state free precession (SSFP) sequence (FOV, $380 \mathrm{~mm}$; matrix, $179 \times 160$; TE, $1.3-1.5 \mathrm{~ms}$; TR, 2.5-2.9 ms automatically determined by the MR computer; FA, $60^{\circ}$; and BW, $2123 \mathrm{~Hz}$ ). 


\section{T2 mapping}

T2 mapping was obtained in the same slice position used for tagging imaging by means of a multi-TE fast spin echo (FSE) sequence. Two different TEs were used: 16-16.5 and $100 \mathrm{~ms}$ (FOV, $380 \mathrm{~mm}$; matrix, $179 \times 224$; TR, 2-3 heartbeats; FA, $90^{\circ}$; BW, $354 \mathrm{~Hz}$; parallel imaging factor, 2 ; NSA, 2; and number of start-up echoes, 3 ).

\section{LGE}

LGE was performed $10 \mathrm{~min}$ after administering $0.1 \mathrm{mmol} /$ $\mathrm{kg}$ of gadolinium-DTPA (Magnevist, Bayer Schering
Pharma AG, Berlin, Germany). LGE was obtained with a three-dimensional (3D) segmented inversion recovery gradient echo sequence with a $10-\mathrm{mm}$ thickness and $5-\mathrm{mm}$ overlap (FOV, $350 \mathrm{~mm}$; matrix, $179 \times 256$; TE, $1.35 \mathrm{~ms}$; TR, $4.5 \mathrm{~ms}$; FA, 15 ; BW, $261.9 \mathrm{~Hz}$; parallel imaging factors, 2 ; and stack, 2). The inversion time (TI) was adjusted to the normal myocardium using TI scout imaging.

Image analysis for tagging imaging (Fig. 1)

The open source software inTag (www.creatis.insa-lyon.fr/ inTag/) was used to analyze the tagging images. Motion estimation of this software is based on the sine wave modeling
Fig. 1 Representative tagging images and T2 mapping. An image at the end of the diastolic phase (i.e., an initial image in the cardiac cycle of tagging imaging) is shown in $\mathbf{a}$, while b shows an image at the peak systolic phase. The image at the mid-ventricle level was divided into four segments: the anterior, lateral, inferior, and septal walls (c). A circumferential strain map is superimposed on the tagging image and is shown in d (The results are also shown in Fig. 2). An original image (e) and a color T2 map (f) are also shown. A diffuse increase in $\mathrm{T} 2$ value is indicated with a purple color
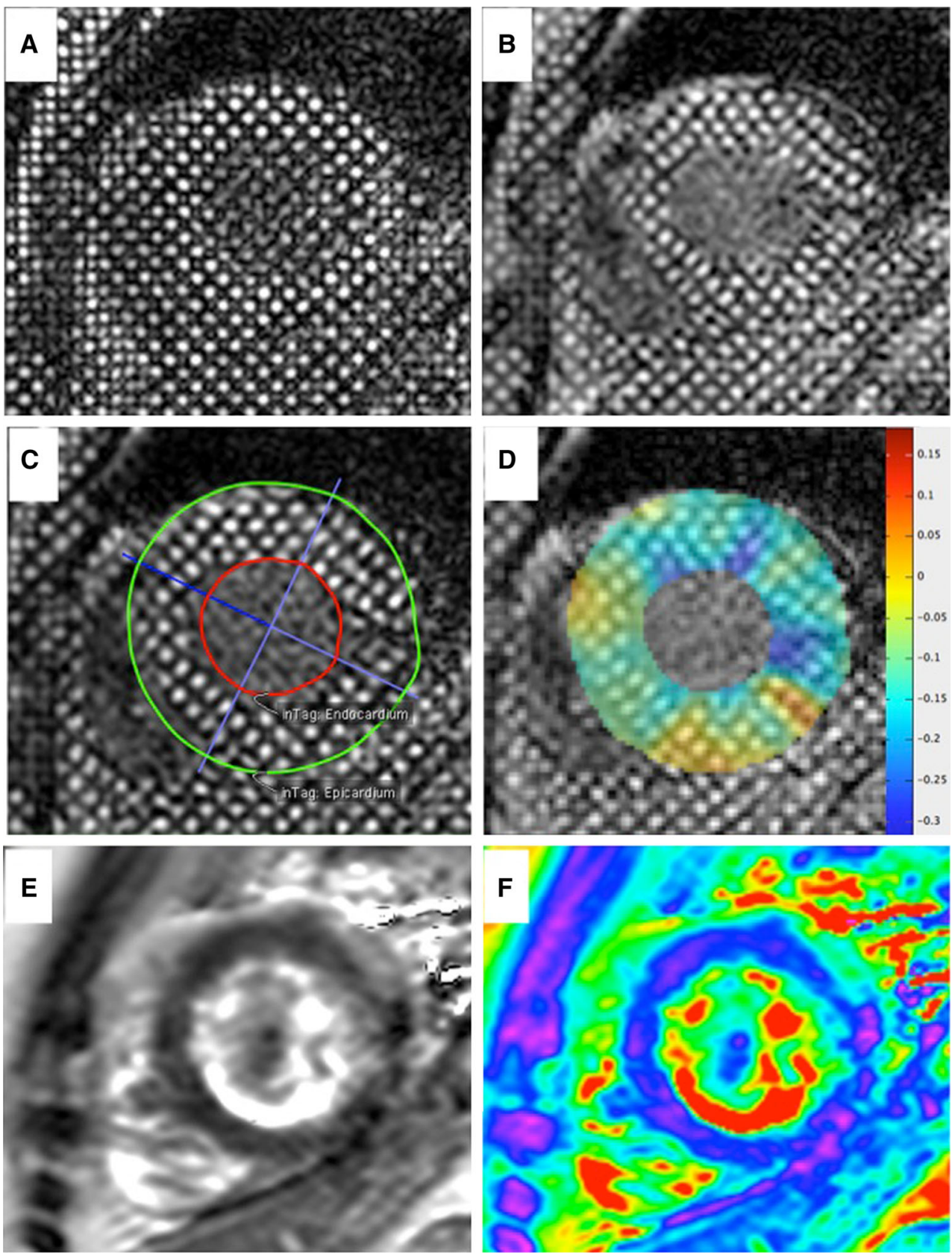
approach [18]. The slice at the mid-ventricle level was selected for the analysis, and the LV myocardium was divided into four segments: the anterior, lateral, inferior, and septal walls. The definition of the anterior junction of both ventricles was set manually on the slice. The contours of the endomyocardium and epimyocardium were traced automatically. We did not divide the myocardial layers in this study (i.e., we adopted "1" as the number of myocardial layers).

\section{Statistical analysis}

Data are presented as mean and standard deviation (SD) for continuous variables and as absolute number for categorical variables. We used analysis of variance (ANOVA) or the Welch test to compare continuous variables and Fisher's exact test to compare categorical variables. The Pearson test was employed to analyze correlations. Statistical analyses of the data were performed using JMP software (version 9.0, SAS Institute, Inc., Cary, NC, USA). A $P$ value $<0.05$ was considered to indicate a significant difference.

We conducted the following analyses:

1. We evaluated the maximum value of the circumferential strain $\left(\mathrm{Ecc}^{*}\right)$ and compared the Ecc* values of DCM and NML groups.

2. We evaluated the time point of Ecc* (Time*) in each segment to determine dyssynchrony, and we calculated the coefficient of variation (CVtime*) using the following equation; CVtime* $(\%)=100 \times($ SD of Time* $/$ mean Time* ${ }^{*}$. We compared CVtime* between DCM and NML groups (Fig. 2).

3. We constructed time-Ecc curves and described their abnormalities following visual inspection.

4. We analyzed the correlation of Ecc* and left ventricular ejection fraction (LVEF) calculated from cine imaging on CMR using the Simpson method.

5. We evaluated $\mathrm{T} 2$ values from $\mathrm{T} 2$ mapping and the area of LGE in DCM, and we analyzed the correlation between Ecc* and $\mathrm{T} 2$ value. The mean $\mathrm{T} 2$ value was measured when we set the region of interest (ROI) to cover the entire myocardium. The LGE-positive area was considered that showing a higher signal intensity with mean +2 SD of normal (null) myocardium and was expressed as a percentage of the LGE-positive area in the entire myocardium.

\section{Results}

Patient characteristics are summarized in Table 1. The values of age, LV end-systolic volume (LVESV), and LVEF were higher in DCM patients than in the NML group. The value of LV end-diastolic volume (LVEDV)

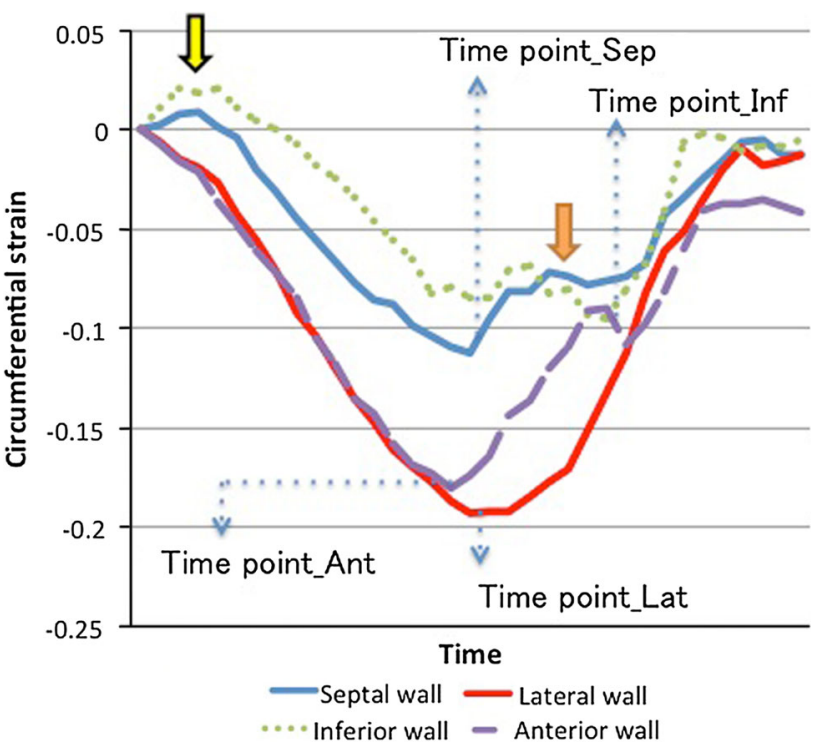

Fig. 2 Time-strain curves obtained for a 28-year-old male DCM patient with a left ventricle ejection fraction (LVEF) of $30.3 \%$. Circumferential strain is plotted against time in the septal (blue line), lateral (red line), inferior (green dotted line), and anterior (purple dashed line) walls. The yellow arrow shows pre-systolic extension, and the orange arrow indicates systolic stretching. Each time point that produced a peak strain in each segment is also indicated. The CVtime* was calculated from these time points

Table 1 Characteristics of normal controls and DCM patients

\begin{tabular}{llll}
\hline & NML $(n=15)$ & DCM $(n=12)$ & $P$ value \\
\hline Age (years) & $28.5 \pm 3.1$ & $48.9 \pm 3.5$ & 0.0024 \\
Gender (n) (m:f) & $15: 0$ & $7: 5$ & 0.0098 \\
LVEDV (ml) & $139 \pm 15.8$ & $193.2 \pm 17.7$ & 0.07 \\
LVESV (ml) & $53.3 \pm 16.1$ & $148.6 \pm 18.0$ & 0.0048 \\
LVEF (\%) & $61.8 \pm 1.7$ & $26.4 \pm 2.2$ & $<0.0001$ \\
BNP (pg/ml) & & $201.1 \pm 281.8$ &
\end{tabular}

$B N P$ B-type natriuretic peptide, DCM dilated cardiomyopathy, $L V E D V$ left ventricle end-diastolic volume, $L V E F$ left ventricle ejection fraction, $L V E S V$ left ventricle end-systolic volume, $N M L$ normal controls

was higher, but the difference was not statistically significant $(P=0.07)$. The mean of the B-type natriuretic peptide (BNP) values was $201.1 \mathrm{pg} / \mathrm{ml}$.

The results of tagging imaging and strain derived from tagging imaging are summarized in Table 2 . The values of Ecc* in NML and DCM groups were $-23.5 \pm 1.0$ and $12.7 \pm 1.2$, respectively, and were significantly decreased in DCM group $(P<0.0001)$. The values for CVtime* in NML and DCM groups were $4.5 \pm 1.4$ and $15.2 \pm 1.5$, respectively, and dyssynchrony was significantly observed in DCM patients $(P=0.002)$.

The time-ECC curves indicated a finding of pre-systolic extension in $6(50 \%)$ DCM patients and systolic stretch in 
Table 2 The results of cardiovascular magnetic resonance imaging

\begin{tabular}{llll}
\hline & $\begin{array}{l}\text { NML } \\
(n=15)\end{array}$ & $\begin{array}{l}\text { DCM } \\
(n=12)\end{array}$ & $P$ value \\
\hline MRI & $3.0 \mathrm{~T}$ & $1.5 \mathrm{~T}$ & \\
Global Ecc* (\%) & $-23.5 \pm 1.0$ & $-12.7 \pm 1.2$ & $<0.0001$ \\
Anterior wall & $-23.6 \pm 1.4$ & $-13.7 \pm 1.6$ & \\
Lateral wall & $-25.7 \pm 1.0$ & $-17.0 \pm 1.1$ & \\
Inferior wall & $-24.1 \pm 1.3$ & $-10.3 \pm 1.4$ & \\
Septal wall & $-20.6 \pm 1.2$ & $-9.5 \pm 1.3$ & \\
CVtime* (\%) & $4.5 \pm 1.4$ & $15.2 \pm 1.5$ & 0.0002 \\
Pre-systolic extension & 0 & $6(50 \%)$ & 0.0031 \\
$\quad$ (n) & & & \\
Systolic stretch (n) & 0 & $10(83.3 \%)$ & $<0.0001$ \\
T2 value (ms) & NA & $64.5 \pm 7.0$ & \\
LGE (\%) & NA & $9.0 \pm 13.3$ & \\
\hline
\end{tabular}

CVtime* coefficient of variation for the time of peak circumferential strain among the myocardial segments, $D C M$ dilated cardiomyopathy, $E C C^{*}$ peak circumferential strain, $L G E$ late gadolinium enhancement, $M R I$ magnetic resonance imaging, $N A$ not assessable, $N M L$ normal controls

$10(83.3 \%)$ DCM patients. None of the NML patients showed either of these findings.

In all subjects, the value of Ecc* correlated well with the $\operatorname{LVEF}\left(R^{2}=0.88, P=0.042\right)$. Extraction of DCM patient data revealed a clearer correlation $\left(R^{2}=0.90, P<0.0001\right)$ (Fig. 3).

DCM patients had a mean $\mathrm{T} 2$ value of $64.5 \pm 7.0 \mathrm{~ms}$, and the percentage of the LGE area was $9.0 \pm 13.3 \%$. The value of Ecc* correlated moderately with the $\mathrm{T} 2$ value $\left(R^{2}=0.44, P=0.018\right)$ (Fig. 4a) but showed no correlation with LGE $\left(R^{2}=0.28, P=0.072\right)$ (Fig. 4b).

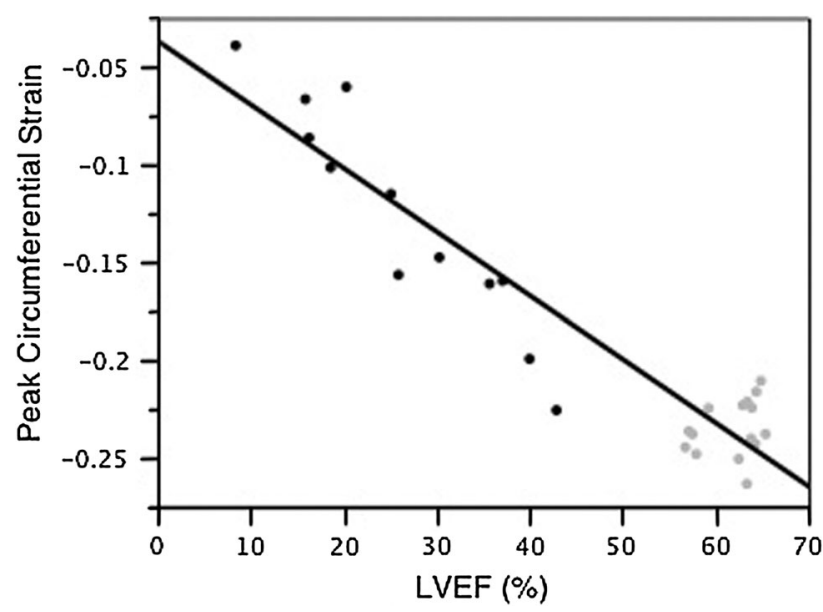

Fig. 3 Scattergrams showing the correlation of peak circumferential strain (Ecc*) with left ventricle ejection fraction (LVEF). Ecc* correlated well with LVEF $\left(R^{2}=0.88, P=0.042\right)$. The black dots represent DCM patients, and gray dots represent normal (NML) controls

\section{Discussion}

Tagging imaging on CMR was introduced in 1988 by Zerhouni et al. [2] and Axel and Dougherty [19, 20]. Black lines or grids are superimposed on the myocardium at the beginning of a cine sequence, and the subsequent deformation throughout the cardiac cycle is noted. Strain (i.e., deformation) refers to the change in shape resulting from contraction, and it is expressed as a percentage of the fractional change in the length $L$ of an elementary myocardial segment in a given direction during the cardiac cycle $(\Delta L / L)$ [21]. Lengthening gives positive strain values, whereas shortening produces negative strain values.

The contraction of the sarcomeres occurs along the myofibers, so active contraction is only longitudinal and circumferential. In contrast, radial thickening is not a primary phenomenon but is merely a consequence of fiber rearrangement. Therefore, circumferential strain is suitable for the analysis of myocardial function and was adopted in our study. Our results showed that Ecc* was lower in DCM patients than in the NML group. The mean Ecc* in NML participants was $-23.5 \%$, which was compatible with previously published results $[1,22,23]$. Del-Canto et al. [23] reported the mean Ecc* at mid-ventricular level in their normal population was $-20.1 \%$ (mean age, 58.8 years). Because our NML controls were younger than DCM group, an age-related decrease in strain might have affected the results. However, the mean value of $\mathrm{Ecc}^{*}$ in DCM was much lower even if we compared it with the results reported by Del-Canto. Peak circumferential strain was previously found to be -5.3 and $5 \%$ in DCM patients $[12,13]$. These results indicated a greater disease severity compared to the present results $(-12.7 \%)$; however, the amount of strain depends on the difference in the degree of severity of myocardial dysfunction in DCM patients, since the previously studied DCM patients showed lower EF values (mean EF of $20.2 \%$ [range 8-30\%] [12] and $16 \%$ [range 8-33\%] [13]) than those measured in the present study (mean EF, $26.4 \%$; ranged 8.4-42.9 \%). In addition, the reduction in Ecc* correlated well with the ejection fraction in this study. Thus, we believe that tagging imaging could show consistency with regard to the severity of the myocardial disease. Strain analysis was sensitive and reliable for detecting myocardial dysfunction. Tagging imaging and strain analysis could provide quantitative values for the assessment of the normal myocardium as well as myocardial dysfunction in DCM patients.

Our results also showed that CVtime* was larger in DCM patients than in NML group. This indicates that wall contraction does not occur simultaneously in each segment, which is probably a consequence of a conduction abnormality and leads to dyssynchrony. Many patients with DCM have conduction abnormalities, especially as they develop 
Fig. 4 Scattergrams showing the correlation of peak circumferential strain $\left(\mathrm{Ecc}^{*}\right)$ with $\mathrm{T} 2$ value and late gadolinium enhancement (LGE). The T2 value was moderately correlated with Ecc* $\left(\mathbf{a} ; R^{2}=0.44, P=0.018\right)$, whereas the LGE area had no correlation with Ecc* (b; $R^{2}=0.28, P=0.072$ )

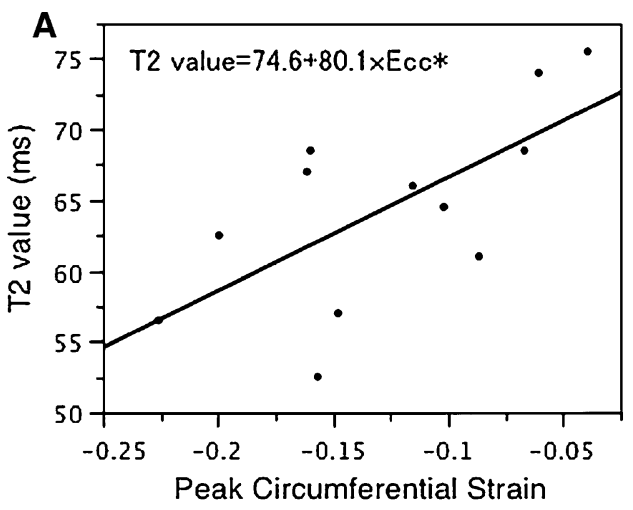

heart failure [24, 25]. Our DCM patients included nine who showed wide QRS (QRS > $100 \mathrm{~ms}$ ) on electrocardiogram (ECG). Among these, 7 (77.8\%) patients had systolic stretch, and five were patients whose cardiologists had diagnosed them with a left bundle branch block (LBBB) or a non-specific conduction abnormality. All of these patients showed a systolic stretch upon tagging imaging. Myocardial dyssynchrony generally occurs as the dysfunction progresses, and it further worsens the function. This dyssynchrony may be expressed in many ways and has been studied for years using ultrasonography procedures such as Doppler tissue imaging and speckle tracking. The SD of the time to peak obtained with MR tagging quantifies dyssynchrony and is altered mainly in LBBBs and ventricular aneurysms [26]. However, several other indexes may be computed from the segmental contraction curve (e.g., strain variance, phase dispersion derived from Fourier analysis, calculation of the amount of energy loss with the "strain delay index"), and no general consensus has yet been made regarding a universal marker for ventricular dyssynchrony [26]. Dyssynchrony is also an important factor when cardiac resynchronization therapy (CRT) is applied in patients with a low LVEF and wide QRS (>120 ms). Therefore, tagging imaging is expected to detect and quantify intraventricular dyssynchrony because higher reproducibility is possible with MRI than with ultrasonography, and reproducibility is not affected by the operator.

We noted two abnormal findings during the visual inspection of time-Ecc curves in our DCM patients. First, six DCM patients had pre-systolic extension. Han et al. [14] reported a finding of "small systolic early contraction" in $50 \%$ of their DCM patients with LBBB. Interestingly, the direction (contraction vs. extension) differed; however, we are unable to explain this discrepancy. Second, 10 DCM patients showed systolic stretch. Although a similar finding was also reported by Jeung et al. [26], they did not disclose its frequency in their population. The same finding was observed in the literature reported by Zwanenburg et al. [27], who also did not mention the frequency. They noted multiple shortening waves, including the systolic stretch, and explained that this was related to interaction of the left and right ventricles in combination with the weak contraction of the early activated septum [27]. In our study, we saw this systolic stretch in patients with relatively preserved function, so we believe this finding is mainly based on the conduction abnormality as well as the ventricle contractile interaction.

The value of Ecc* showed a moderate correlation with T2 value, although it did not correlate with the LGE. To the best of our knowledge, this was the first study to mention the correlation of ECC with T2 value. Essentially, LGE showed an excellent correlation with the myocardial scar pathologically [28], and a myocardium with LGE produced regional dysfunction in ischemic heart disease [29]. In DCM patients, the myocardial scar eventually becomes a factor for myocardial dysfunction; therefore, the presence of LGE on CMR is one of the markers correlated with a poor prognosis or non-response to treatment [30]. However, the frequency of LGE in DCM is estimated to be 28-35\% [9] and will vary according to the population evaluated. Nevertheless, LGE was not seen in all myocardial segments or patients, in spite of their diffuse myocardial dysfunction. Although LGE is an important contributor, other factors may explain the diffuse myocardial dysfunction in the absence of scarring. Chronic inflammation is viewed as one of the causes of DCM and plays an important role in disease progression. Inflammatory cells were detected from pathology of the diseased myocardium of DCM patients [31]. Areas of inflammation or edema will show higher T2 values on MRI, so detection of myocardial damage is possible by measuring T2 [32]. The T2 measurement technique has been used in ischemic heart disease [33], but its usefulness has not been established in DCM patients. In the present study, we adopted a T2 mapping technique and identified a correlation between Ecc* and T2 value. Our findings indicate that myocardial dysfunction occurs not only in areas of myocardial scarring, but also in areas with edema, suggesting that inflammation affects myocardial function. Further examination is needed to confirm this idea. 
In summary, strain derived from tagging imaging provides useful objective and quantitative indexes that are superior to the simple visual assessment of $\mathrm{LV}$ motion abnormalities. The decrease in circumferential strain was coincident with the myocardial inflammation. Tagging imaging is also effective because it is a widely available method and does not require contrast material.

\section{Limitations}

The number of DCM patients in our study was small. One reason for this was that tagging imaging is sensitive to arrhythmia [26], so these examinations were not performed on patients with arrhythmias. We also did not evaluate the clinical usefulness of tagging imaging in DCM, such as the predictability of the prognosis, because of the small number of patients.

This study used a 1.5-T scanner for DCM patients and 3.0-T MRI for the NML group. The fading effect diminished the tag lines in the diastolic phase, especially with 1.5 $\mathrm{T}$. We analyzed the tag only in the systolic phase in order to avoid the fading effect; thus, we believe the influence of different MR equipment was small. In addition, the strength of the magnetic field does not theoretically affect the value of the strain.

Our T2 mapping technique adopted two different TEs (16.0-16.5 and $100 \mathrm{~ms}$ ) because of the limitations of our machine. Although a multi-echo method with more than three TEs provides more accurate $\mathrm{T} 2$ values, it requires long scan times, which limits its feasibility for use in clinical settings. However, a recent paper using an MR scanner with a higher magnetic field strength and a singleshot SSFP readout sequence with three TEs showed highly accurate and reproducible results [34].

In conclusions, strain derived from tagging imaging revealed myocardial dysfunction and dyssynchrony of the contraction in DCM and was correlated with myocardial $\mathrm{T} 2$ value.

Acknowledgments The author (AKK) received an award from the Japanese Society for Magnetic Resonance in Medicine and Bayer Schering Pharma. This study was partially funded by this award. I would like to express my gratitude to my family for the support of my fellowship and to Professor Gabriel P. Krestin at Erasmus Medical Center for his insightful comments and suggestions.

Conflict of interest None.

\section{References}

1. Moore CC, McVeigh ER, Zerhouni EA (2000) Quantitative tagged magnetic resonance imaging of the normal human left ventricle. Top Magn Reson Imaging 11(6):359-371
2. Zerhouni EA, Parish DM, Rogers WJ, Yang A, Shapiro EP (1988) Human heart: tagging with MR imaging - a method for noninvasive assessment of myocardial motion. Radiology 169(1):59-63

3. Croisille P, Moore CC, Judd RM, Lima JA, Arai M, McVeigh ER, Becker LC, Zerhouni EA (1999) Differentiation of viable and nonviable myocardium by the use of three-dimensional tagged MRI in 2-day-old reperfused canine infarcts. Circulation 99(2):284-291

4. Gotte MJ, van Rossum AC, Twisk JWR, Kuijer JPA, Marcus JT, Visser CA (2001) Quantification of regional contractile function after infarction: strain analysis superior to wall thickening analysis in discriminating infarct from remote myocardium. J Am Coll Cardiol 37(3):808-817

5. Kuijpers D, Ho KY, van Dijkman PR, Vliegenthart R, Oudkerk M (2003) Dobutamine cardiovascular magnetic resonance for the detection of myocardial ischemia with the use of myocardial tagging. Circulation 107(12):1592-1597

6. Luk A, Ahn E, Soor GS, Butany J (2009) Dilated cardiomyopathy: a review. J Clin Pathol 62(3):219-225

7. Jefferies JL, Towbin JA (2010) Dilated cardiomyopathy. Lancet 375(9716):752-762

8. Jeserich M, Foll D, Olschewski M, Kimmel S, Friedrich MG, Bode C, Geibel A (2012) Evidence of myocardial edema in patients with nonischemic dilated cardiomyopathy. Clin Cardiol 35(6):371-376

9. Assomull RG, Prasad SK, Lyne J, Smith G, Burman ED, Khan M, Sheppard MN, Poole-Wilson PA, Pennell DJ (2006) Cardiovascular magnetic resonance, fibrosis, and prognosis in dilated cardiomyopathy. J Am Coll Cardiol 48(10):1977-1985

10. McCrohon JA, Moon JC, Prasad SK, McKenna WJ, Lorenz CH, Coats AJ, Pennell DJ (2003) Differentiation of heart failure related to dilated cardiomyopathy and coronary artery disease using gadolinium-enhanced cardiovascular magnetic resonance. Circulation 108(1):54-59

11. MacGowan GA, Shapiro EP, Azhari H, Siu CO, Hees PS, Hutchins GM, Weiss JL, Rademakers FE (1997) Noninvasive measurement of shortening in the fiber and cross-fiber directions in the normal human left ventricle and in idiopathic dilated cardiomyopathy. Circulation 96(2):535-541

12. Nelson GS, Curry CW, Wyman BT, Kramer A, Declerck J, Talbot M, Douglas MR, Berger RD, McVeigh ER, Kass DA (2000) Predictors of systolic augmentation from left ventricular preexcitation in patients with dilated cardiomyopathy and intraventricular conduction delay. Circulation 101(23):2703-2709

13. Young AA, Dokos S, Powell KA, Sturm B, McCulloch AD, Starling RC, McCarthy PM, White RD (2001) Regional heterogeneity of function in nonischemic dilated cardiomyopathy. Cardiovasc Res 49(2):308-318

14. Han Y, Chan J, Haber I, Peters DC, Zimetbaum PJ, Manning WJ, Yeon SB (2010) Circumferential myocardial strain in cardiomyopathy with and without left bundle branch block. J Cardiovasc Magn Reson 12:2

15. Kanzaki H, Nakatani S, Yamada N, Urayama S, Miyatake K, Kitakaze M (2006) Impaired systolic torsion in dilated cardiomyopathy: reversal of apical rotation at mid-systole characterized with magnetic resonance tagging method. Basic Res Cardiol 101(6):465-470

16. Russel IK, Gotte MJ, de Roest GJ, Marcus JT, Tecelao SR, Allaart CP, de Cock CC, Heethaar RM, van Rossum AC (2009) Loss of opposite left ventricular basal and apical rotation predicts acute response to cardiac resynchronization therapy and is associated with long-term reversed remodeling. J Card Fail 15(8):717-725

17. Elliott P, Andersson B, Arbustini E, Bilinska Z, Cecchi F, Charron P, Dubourg O, Kuhl U, Maisch B, McKenna WJ, Monserrat L, Pankuweit S, Rapezzi C, Seferovic P, Tavazzi L, Keren A (2008) Classification of the cardiomyopathies: a position 
statement from the European Society Of Cardiology Working Group on Myocardial and Pericardial Diseases. Eur Heart J 29(2):270-276

18. Arts T, Prinzen FW, Delhaas T, Milles JR, Rossi AC, Clarysse P (2010) Mapping displacement and deformation of the heart with local sine-wave modeling. IEEE Trans Med Imaging 29(5): 1114-1123

19. Axel L, Dougherty L (1989) Heart wall motion: improved method of spatial modulation of magnetization for MR imaging. Radiology 172(2):349-350

20. Axel L, Dougherty L (1989) MR imaging of motion with spatial modulation of magnetization. Radiology 171(3):841-845

21. Shehata ML, Cheng S, Osman NF, Bluemke DA, Lima JA (2009) Myocardial tissue tagging with cardiovascular magnetic resonance. J Cardiovasc Magn Reson 11:55

22. Kuijer JP, Marcus JT, Gotte MJ, van Rossum AC, Heethaar RM (2002) Three-dimensional myocardial strains at end-systole and during diastole in the left ventricle of normal humans. J Cardiovasc Magn Reson 4(3):341-351

23. Del-Canto I, Lopez-Lereu MP, Monmeneu JV, Croisille P, Clarysse P, Chorro FJ, Bodi V, Moratal D (2013) Characterization of normal regional myocardial function by MRI cardiac tagging. J Magn Reson Imaging

24. Meinertz T, Hofmann T, Kasper W, Treese N, Bechtold H, Stienen U, Pop T, Leitner ER, Andresen D, Meyer J (1984) Significance of ventricular arrhythmias in idiopathic dilated cardiomyopathy. Am J Cardiol 53(7):902-907

25. Akar FG, Tomaselli GF (2005) Conduction abnormalities in nonischemic dilated cardiomyopathy: basic mechanisms and arrhythmic consequences. Trends Cardiovasc Med 15(7):259-264

26. Jeung MY, Germain P, Croisille $P$, El ghannudi S, Roy C, Gangi A (2012) Myocardial tagging with MR imaging: overview of normal and pathologic findings. Radiographics 32(5):1381-1398

27. Zwanenburg JJ, Gotte MJ, Marcus JT, Kuijer JP, Knaapen P, Heethaar RM, van Rossum AC (2005) Propagation of onset and peak time of myocardial shortening in time of myocardial shortening in ischemic versus nonischemic cardiomyopathy: assessment by magnetic resonance imaging myocardial tagging. J Am Coll Cardiol 46(12):2215-2222

28. Kim RJ, Chen EL, Lima JA, Judd RM (1996) Myocardial GdDTPA kinetics determine MRI contrast enhancement and reflect the extent and severity of myocardial injury after acute reperfused infarction. Circulation 94(12):3318-3326

29. Kim RJ, Wu E, Rafael A, Chen EL, Parker MA, Simonetti O, Klocke FJ, Bonow RO, Judd RM (2000) The use of contrastenhanced magnetic resonance imaging to identify reversible myocardial dysfunction. N Engl J Med 343(20):1445-1453

30. Lehrke S, Lossnitzer D, Schob M, Steen H, Merten C, Kemmling H, Pribe R, Ehlermann P, Zugck C, Korosoglou G, Giannitsis E, Katus HA (2011) Use of cardiovascular magnetic resonance for risk stratification in chronic heart failure: prognostic value of late gadolinium enhancement in patients with non-ischaemic dilated cardiomyopathy. Heart 97(9):727-732

31. Maehashi N, Yokota Y, Takarada A, Usuki S, Maeda S, Yoshida H, Sugiyama T, Fukuzaki H (1991) The role of myocarditis and myocardial fibrosis in dilated cardiomyopathy. Analysis of 28 necropsy cases. Jpn Heart J 32(1):1-15

32. Giri S, Chung YC, Merchant A, Mihai G, Rajagopalan S, Raman SV, Simonetti OP (2009) T2 quantification for improved detection of myocardial edema. J Cardiovasc Magn Reson 11:56

33. Verhaert D, Thavendiranathan P, Giri S, Mihai G, Rajagopalan S, Simonetti OP, Raman SV (2011) Direct T2 quantification of myocardial edema in acute ischemic injury. JACC Cardiovasc Imaging 4(3):269-278

34. von Knobelsdorff-Brenkenhoff F, Prothmann M, Dieringer MA, Wassmuth R, Greiser A, Schwenke C, Niendorf T, SchulzMenger J (2013) Myocardial T1 and T2 mapping at 3 T: reference values, influencing factors and implications. J Cardiovasc Magn Reson 15(1):53 